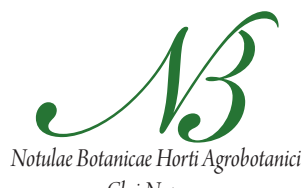

Cluj-Napoca

\title{
The Influence of the Developmental Stage and Culture Medium upon the Somatic Embryo Induction in Quercus petraea
}

\author{
Adrian I. TIMOFTE, Simona VICAȘ \\ University of Oradea, Faculty of Environmental Protection, 26 Gen. Magheru St., 410058 \\ Oradea,Romania; adi_timofte@yahoo.com,svicas@uoradea.ro
}

\begin{abstract}
The somatic embryogenesis is an advanced method for clonal propagation and a useful tool for ex situ conservation of genetic resources. In this paper an experimental device was designed, composed of: one oak species (Quercus petraea), one provenance, four types of explants (developmental stages of the zygotic embryo) and four variants of culture medium. It was released the two-way statistical analyses of the two analyzed factors affecting the embryo induction efficiency: the stage of explant and culture medium (4 stages $\mathrm{x}$ 4 culture media). The most important factor affecting the efficiency of somatic embryogenesis was found to be the developmental stage of the zygotic embryos used as juvenile explants. The high embryogenic ability of young zygotic embryos and low embryogenic ability of maturing zygotic ones showed the strong positive relationship between somatic embryo induction and accumulation of reserve nutrients in cotyledons. The best results were obtained with the youngest embryos (stage 1), with a linear decrement towards the stage 4. The embryo induction efficiency ranged between $44 \%$ and $10 \%$ in $Q$. petraea. Two nutritive culture media and two growth hormone combinations were tested. The effect of culture medium proved to be totally insignificant, besides the differences observed among the variants cultivated on different media. The situation was the same as well for the nutrients as for growth regulators.
\end{abstract}

Keywords: developmental stage, embryogenic induction, Quercus petraea, somatic embryogenesis

\section{Introduction}

The sessile oak (Q. petraea) is among the most important broadleaved forest tree species in Europe. In Romania, sessile oak occupy approximately $10 \%$ of the forest area, having a high ecological and economic value. The in vitro plant regeneration by somatic embryogenesis is a highly conservative process and a potential source of genetic variability.

The use of somatic embryogenesis in oak clonal micropropagation is preferable in the applications concerning somaclonal variation and selection, because the plants regenerated from somatic embryos usually have monocellular origin and consist of clones of long-term culture, submitted of in vitro selection procedures. This advanced method of plant propagation was also applied in the ex situ conservation of genetic resources, using the cryopreservation techniques (Jorgensen, 1991, 1993; Tutkova and Wilhelm, 1999; Pijut et al., 2007; Fernandes et al., 2008; Vengadesan and Pijut, 2009a, 2009b).

The somatic embryogenesis of oak, as an alternative to cutting propagation, provides the possibility of mass production of cotyledonary embryos, which can be either cryopreserved, or maintained by long-term culture as stable embryogenic somaclones (Wilhelm et al., 1996, 2000).
The aim of this study was to assess the influence of the developmental stage and culture medium on the somatic embryo induction in Quercus petraea acorns.

\section{Materials and methods}

Plant material: The immature acorns of $Q$. petraea harvested from Mihăieşti-Argeş at 4 different dates which were dissected and used as sources of explants. The explants were represented by immature zygotic embryos in different developmental stages or fragments of more advanced embryos. The developmental stages of zygotic embryos have been defined and correlated with the morphological characteristics of acorns (Palada-Nicolau and Hausman, 2001):

Stage 1: acorn has a diameter of 6-8 $\mathrm{mm}$ and the cup covers it almost completely, the zygotic embryo of 1-1.2 $\mathrm{mm}$ is inside the acorn in an early cotyledonous stage;

Stage 2: acorn has a diameter of about $10 \mathrm{~mm}$ and is at the level of the cup, the zygotic embryo of 2-2.4 mm has a translucid but thicker cotyledons, the endosperm, well represented, is in a cell stage;

Stage 3: acorn is elongated, having dimensions of 10/ $12 \mathrm{~mm}$ and exceeds the level of the cup, the embryo which is also elongated $(5 / 3-8 / 3 \mathrm{~mm})$ has thickened;

Stage 4: acorn of 12-13/16-18 $\mathrm{mm}$ is only half covered by the cup, the embryo of $10 / 7 \mathrm{~mm}$ has thick and opaque 
386

cotyledons and obvious meristematic areas (apical and radicular), and is completely without endosperm.

\section{Culture medium}

Four media were tested for the induction of embryogenesis, representing combinations between two nutrient recipes (1/2 MS - Murashige, Skoog, 1962) and DCR (Gupta, Durzan, 1985) and two combinations of growth regulators: QE1: $1 / 2 \mathrm{MS}$ with $10 \mu \mathrm{M} / 12,4-\mathrm{D}$ and $2 \mu \mathrm{M} / 1$ BAP; QE2: 1/2MS with $5 \mu \mathrm{M} / 1 \mathrm{NAA}-5 \mu \mathrm{M} / \mathrm{l} \mathrm{BAP}$; QE3: DCR with $10 \mu \mathrm{M} / 1$ 2,4-D and $2 \mu \mathrm{M} / \mathrm{l}$ BAP; QE4: DCR with $5 \mu \mathrm{M} / 1$ NAA - $5 \mu \mathrm{M} / 1$ BAP. After two subcultures on this medium, a hormone-free $1 / 2 \mathrm{MS}$ medium was used for the proliferation of embryogenic structures.

\section{Structure of the experiment}

4 developmental stages of explants, 4 culture media (represented by: 2 variants of nutritive medium: 1/2 MS (Murashige-Skoog, 1962) and DCR (Gupta, Durzan, 1985 ) and 2 variants of growth regulator combinations, for each nutritive medium). In order to establish the effect of culture medium upon the efficiency of somatic embryo induction, comparisons were made between nutritive media (QE1 + QE2, versus QE3 + QE4), on one hand, and between the two combinations of growth regulators $(\mathrm{QE1}$ $+\mathrm{QE3}$, versus QE2 + QE4), on the other hand. Three replications consisting of ten explants have been counted for each parameter.

\section{Statistical analyses}

A two ways ANOVA in randomised blocks design, for experiments in three replications was used in order to validate the results of the oak somatic embryogenesis experiments, in which the main parameters were explant stages and culture media. The statistical validation of the differences between the experimental variants was made by using the multiple range test (Duncan). These tests allowed the objective comparison of all differences between every two variants.

Besides embryogenesis, other reactions of the explants to the culture conditions were observed: callogenesis, rhyzogenesis and germination.

\section{Results and discussions}

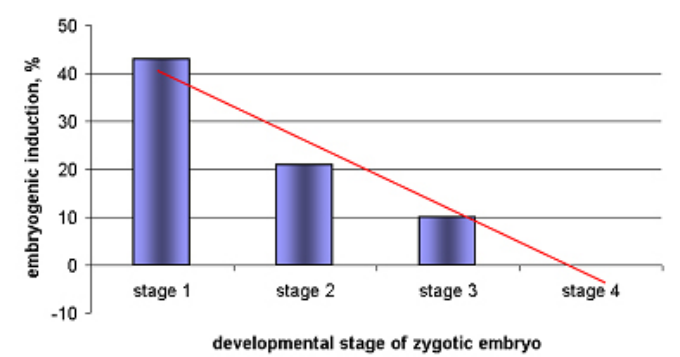

Fig. 1. The effect of explant type upon the embryogenic ability, in $Q$. petraea
The embryogenic ability decreased constantly from the youngest to the more developed zygotic embryos used as explants (Fig. 1).

The effect of tested culture media on the embryogenesis proved to be insignificant for the same type of explants (development stage), based on the differences observed among the variants cultivated on different media, irrespective of the combinations of nutrients and growth regulators used in the study.

Thus, the nutrient medium had no significant effect on the embryogenic capacity of explants, both tested culture

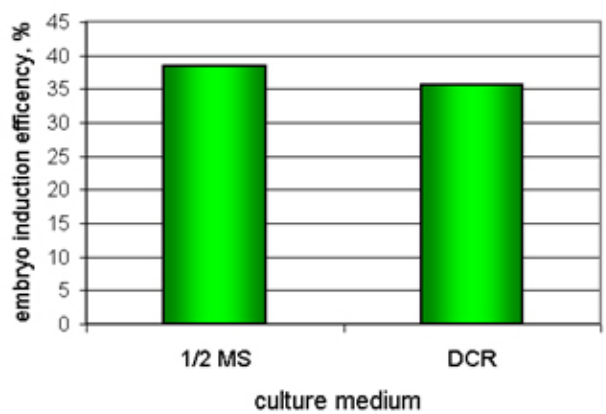

Fig. 2. The effect of culture medium on the embryo induction efficency in Q.petraea

media being capable of supporting embryogenesis, if the other conditions (proper development stage of embryos) were met (Fig. 2).

Regarding the role of growth regulators, the comparisons between the embryogenic induction efficiency of the media with auxin 2,4-D - 2,4-dichlorophenoxiacetic acid (QE1 and QE3) and those with NAA - a-naphtyl-acetic acid (QE2 and QE4) showed that the two combinations of auxins and cytokinins didn't have a significant effect on

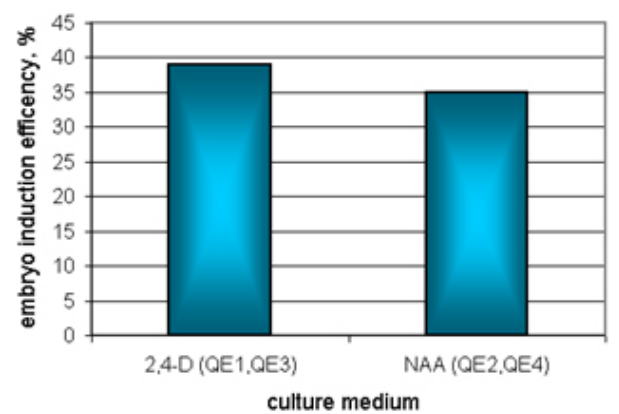

Fig. 3. The effect of auxin type on the embryo induction efficency in Q. petraea

the embryogenic capacity, the differences between the results of the efficiency for the two types of media being not significant (Fig. 3).

The statistical analyses showed no significant differences between the results obtained on different culture media for the same development stage and also for the interactions development stage $\mathrm{x}$ medium. However highly sig- 
Tab. 1. The effect of the developmental stage of explant on the rate of embryogenic induction in Q. petraea (Duncan test)

\begin{tabular}{ccc}
\hline Stage of explants (zygotic embryos) & Mean value & Significance of the differences* \\
S1 & 0.814 & M \\
S2 & 0.590 & $\mathrm{~N}$ \\
S3 & 0.422 & $\mathrm{P}$ \\
S4 & 0.311 & $\mathrm{Q}$ \\
\hline
\end{tabular}

${ }^{*}$ Differences among any variants followed by one common letter are not significant DS $5 \%$ for stage: $0.086-0.093$

nificant differences between the embryogenic induction of the tested development stages were found (Tab. 1.)

The study also showed that the composition of the tested culture medium was not a limitative factor, media with different nutritive compositions and auxin/cytokinin ratios of 5/1 (QE1, QE3) and 1/1 (QE2, QE4) being equally suitable for the induction of somatic embryogenesis.

\section{Conclusions}

The results of this study revealed the importance of harvesting the acorns for somatic embryogenesis in early stages; when this is not possible, a significantly higher number of explants is needed in order to secure the desired number of propagated individuals.

\section{References}

Fernandes P, Rodriguez E, Pinto G, Roldan-Ruiz I, De Loose M, Santos C (2008). Cryopreservation of Quercus suber somatic embryos by encapsulation-dehydration and evaluation of genetic stability. Tree Physiology 28(12):1841-1850.

Gupta PK, Durzan DJ (1985). Shoot multiplication from mature trees of Douglas fir (Pseudotsuga menziesii) and Sugar pine (Pinus lambertiana). Plant Cell Rep. 4:177-179.

Jorgensen J (1991). Cryopreservation of haploid embryos of Quercus petraea, Fagus sylavtica and somatic embryos of Aesculus hippocastanum. In: Ahuja (Ed.), Woody Plant Biotechnology, pp. 355-356. Plenum Press, New York.
Jorgensen J (1993). Embryogenesis in Quercus petraea. Ann Sci For 50(1):344s-350s.

Murashige T, Skoog F (1962). A revised medium for rapid growth and bioassays with tobacco tissue cultures. Physiol. Plant 15:473-497.

Palada-Nicolau M, Hausman J-F (2001). Comparison between somatic and zygotic embryo development in Quercus robur L. Plant Biosystems 135(1):1-9.

Pijut PM, Woeste KE, Vengadesan G, Michler CH (2007) Technological advances in temperate hardwood tree improvement including breeding and molecular marker applications. In vitro Cell Dev Biol Plant 43:283-303.

Tutkova M, Wilhelm E (1999). Cryopreservation of Quercus robur somatic embryos. In vitro Cell. Dev. Biol. Plant 35:180.

Vengadesan G, Pijut PM (2009a). Somatic embryogenesis and plant regeneration of northern red oak (Quercus rubra L.). Plant Cell Tiss Organ Cult 97:141-149.

Vengadesan G, Pijut PM (2009b). In vitro propagation of northern red oak (Quercus rubra L.). In vitro Cell. Dev. Biol. Plant 45:474-482.

Wilhelm E, Burg A, Berenyi M, Endelmann M, Rodler R (1996). Plantlet regeneration via somatic embryogenesis and investigations on Agrobacterium tumefaciens mediated transformation of oak (Quercus robur), p. 125-130. In: Ahuja MR, Boerjan W, Neale DB (Eds.). Somatic Cell Genetics and Molecular Genetics of Trees, Kluwer Press.

Wilhelm E (2000). Somatic embryogenesis in oak (Quercus spp.). In vitro Cell Dev Biol Plant 36:349-357. 